\title{
TAMANHO DE UNIDADE DE AMOSTRA E INTENSIDADE AMOSTRAL PARA ESPÉCIES COMERCIAIS DA AMAZÔNIA
}

\author{
Francisco José de Barros Cavalcanti ${ }^{1}$; Sebastião do Amaral Machado ${ }^{2}$; Roberto Tuyuoshi Hosokawa ${ }^{3}$ \\ ${ }^{1}$ Eng. Florestal, Dr., UFAM, Manaus, AM, Brasil - fjbcavalcanti@ufam.edu.br \\ ${ }^{2}$ Eng. Florestal, PhD, Depto. de Ciências Florestais, UFPR, Curitiba, PR, Brasil, Pesquisador 1A do CNPq - samachado@ufpr.br \\ ${ }^{3}$ Eng. Florestal, PhD, Depto. de Ciências Florestais, UFPR, Curitiba, PR, Brasil, Pesquisador 1B do CNPq - rth@japan.org.br
}

Recebido para publicação: 26/09/2007 - Aceito para publicação: 04/06/2008

\begin{abstract}
Resumo
O objetivo desta pesquisa foi definir a intensidade de amostragem suficiente para atender à exigência atual do Ibama, que limita em 10\% o erro amostral para as variáveis abundância, área basal e volume de árvores adultas, de espécies comerciais em planos de manejo florestal. Para isso, foram simuladas amostragens com diferentes tamanhos de unidades amostrais sobre uma área de 1000 ha localizada no município de Sena Madureira, no estado do Acre, a qual foi inventariada através de enumeração completa (censo florestal), com localização espacial de todas as árvores com diâmetro $\geq 40 \mathrm{~cm}$. A seleção do tamanho das unidades amostrais foi baseada na estabilidade do Coeficiente de Variação, no menor erro amostral, no menor número dimensionado de unidades de amostra e na praticidade da aplicação da metodologia em campo. As intensidades de amostragem definidas, de $11 \%$ para 1 ha, e de $13 \%$ para 2 ha, sugerem a alternativa de estabelecimento de diretrizes de amostragem menos onerosas. Foi proposta a desobrigação do inventário diagnóstico em áreas privadas, tendo em vista que as licenças de exploração são dadas com base apenas no censo florestal. Para as florestas públicas, todavia, foi proposto o estabelecimento de um sistema de amostragem padrão, com custo prefixado e reduzido ou limite de erro maior.

Palavras-chave: Inventário amostral; tamanho de unidades amostrais; Floresta Amazônica.
\end{abstract}

Abstract
Size of sampling unit and sampling intensity for commercial species from the Amazonian Forest. The objective of this research was to define the minimum necessary intensity of sampling to attend to the current legal demand of IBAMA that limits in $10 \%$ the sampling error for the variables: abundance, basal area and volume of adult trees, of commercial species in forest management plans. The selection of the sampling size unit was based on the stability of the Coefficient of Variation, with the minimum acceptable sampling error, with the smaller number of sampling units and regarding the practical applicability of the methodology in the field. The resultant sampling intensities of $11 \%$ with plot of 1 ha, and with $13 \%$, for 2 ha plots, suggest that IBAMA could adopted less onerous alternatives. The non-obligation of a diagnostic inventory was proposed on private areas, given the fact that logging permits issued by this governmental agency are based upon the forest census (100\% inventory). To the public forests, however, it was proposed the establishment of a standard sampling system with pre-established and reduced costs or greater sampling error.

Keywords: Forest inventory; size of sampling units; Amazonian Forest.

\section{INTRODUÇÃO}

O governo brasileiro, através da Lei 11.284, de 2 de março de 2006, para a gestão de florestas públicas, tomou uma importante iniciativa no sentido de promover o uso sustentável das florestas nacionais, estabelecendo três modelos de gestão: unidades de conservação, destinação não-onerosa para o uso comunitário e contratos de concessão florestal. Na mesma lei foi criado o Serviço Florestal Brasileiro, que será gestor das florestas públicas brasileiras.

Entre as atribuições do Serviço Florestal Brasileiro, constam promover e gerenciar a concessão das florestas públicas. O Decreto 6.063, de 2007, estabelece que as concessões se dêem por licitação, sendo vencedora a proposta que maior pontuação obtiver no preço ofertado pelos produtos florestais 
explorados e nos benefícios sociais e ambientais compromissados.

Para a realização de uma licitação, será preciso avaliar a floresta em termos do seu potencial de madeira comercial, de forma que os interessados possam fazer suas ofertas. Nesse sentido, quanto mais exata for a avaliação dos recursos florestais existentes, mais embasadas serão as ofertas.

O Ibama, através da sua Norma Técnica $n^{\circ}$ 01, de 2007 (NT 01/07), determina que os inventários florestais deverão apresentar estimativas de média para a abundância, para a área basal e para o volume com erros amostrais não superiores a $10 \%$ e com $95 \%$ de probabilidade para a população comercial, isto é, considerando as espécies comerciais e o DAP mínimo de $50 \mathrm{~cm}$ para as árvores potencialmente exploráveis.

Queiroz (1977), utilizando o Coeficiente de Variação como critério de seleção de alternativas de sistema de amostragem, avaliando a população arbórea com DAP $\geq 30 \mathrm{~cm}$, na floresta da região de Curuá-Una, no Pará, variou o tamanho da unidade de amostra de $400 \mathrm{~m}^{2}$ a $10.000 \mathrm{~m}^{2}$ e concluiu que, para a variável volume, o tamanho ideal da unidade de amostra foi de $3.200 \mathrm{~m}^{2}$ para a população estudada. Esse mesmo autor definiu também que a distância entre as unidades de amostra e o centro dos conglomerados utilizados para inventários florestais não deveria ser inferior a 50 metros.

Silva (1980), trabalhando na mesma região, definiu o tamanho da unidade de amostra em 0,09 ha para avaliação da regeneração natural da floresta, e o tamanho de 0,25 ha, para a avaliação da população adulta. No referido estudo, o autor considerou como regeneração natural a população com $15 \mathrm{~cm} \leq$ DAP $<45 \mathrm{~cm}$. Como população adulta, o autor considerou as árvores com DAP $\geq 45 \mathrm{~cm}$.

Higuchi et al. (1982), trabalhando na Estação Experimental de Silvicultura Tropical do INPA, no Centro de Apoio da ZF-2, no Distrito Agropecuário da Zona Franca de Manaus, testaram diferentes tamanhos e formas de unidades de amostra, em uma população vegetal 100\% conhecida, a fim de definir o ideal. Para uma população de DAP $\geq 25 \mathrm{~cm}$, os autores selecionaram o tamanho de $37,5 \times 150 \mathrm{~m}$. Na mesma área, Higuchi et al. (1987), comparando a amostragem sistemática com a amostragem aleatória, concluíram pela vantagem da primeira, em função do menor erro amostral.

O objetivo deste trabalho foi definir, através do teste de diferentes tamanhos de unidades de amostra, a intensidade amostral necessária para satisfazer as exigências da NT 01/07 e subsidiar o Serviço Florestal Brasileiro na definição da metodologia de inventários amostrais em florestas públicas passíveis de concessão florestal. Para tanto, foi considerado um erro amostral máximo de 10\% sobre a média da abundância, da área basal e do volume das árvores das espécies comerciais passíveis de exploração, sob uma probabilidade de $95 \%$ de que a média real não ultrapasse o intervalo de confiança da média verdadeira.

Uma vez que as árvores atualmente pertencentes à classe de diâmetro imediatamente inferior à comercial serão aquelas que comporão a maioria da população a ser explorada no próximo ciclo de corte e considerando a expectativa de uma intensidade amostral muito alta para atender a todas as exigências da NT 01/07, a população considerada para a análise foi a de árvores a partir de DAP $\geq 40 \mathrm{~cm}$.

\section{MATERIAL E MÉTODOS}

\section{Área de estudo}

A área de estudo é composta por duas fazendas contíguas, denominadas de São Jorge 1 e São Jorge 2, que perfazem 7.885 hectares, localizadas ao norte do município de Sena Madureira, no estado do Acre. Nela foi realizado o censo florestal em 1.000 hectares, subdivididos em 10 unidades de trabalho de 100 hectares cada uma. Esses 1000 ha serviram de base para a presente pesquisa.

O clima da área é classificado como equatorial quente úmido, apresentando uma precipitação pluviométrica média entre 2000 e $2250 \mathrm{~mm}$ anuais, com longa estação chuvosa.

A área possui variabilidade quanto às suas características geológicas, hidrográficas e geomorfológicas. Encontram-se várias classes de solo, que se apresentam bem distribuídas desde as margens do Rio Iaco, onde predominam os solos hidromórficos, até às nascentes dos pequenos igarapés, onde são observados principalmente os argissolos. De acordo com o Zoneamento Ecológico-Econômico do Acre, citado por Funtac (2002), encontram-se nas unidades de mapeamento as seguintes associações: nas áreas de terra firme ocorre o Argissolo Vermelho Distrófico latossólico textura argilosa + Latossolo Amarelo Distrófico argissólico textura argilosa floresta aberta relevo suave ondulado a ondulado. Também ocorre o Argissolo Amarelo Eutrófico plíntico textura argilosa floresta aberta relevo ondulado + Argissolo Amarelo 
Eutrófico plíntico textura muito argilosa floresta densa e floresta aberta relevo suave ondulado. Nas áreas aluviais ocorre o Alissolo Crômico argilúvico típico textura média + Argissolo Amarelo distrófico textura argilosa floresta aberta relevo plano a suave ondulado e o Gleissolo Hálico Ta eutrófico textura argilosa + Neossolo Flúvico Ta eutrófico textura indiscriminada floresta aberta aluvial relevo plano (RADAMBRASIL, 1976).

A vegetação da área é predominantemente composta por Floresta Aberta, cujas formas mais dominantes são a Floresta Tropical Aberta com Palmeiras e a Floresta Tropical Aberta com Bambu (taboca).

\section{Censo florestal}

Para a realização do censo florestal, ou inventário a $100 \%$, foram abertas picadas paralelas na direção norte-sul, distantes $50 \mathrm{~m}$ entre si. Foram registradas as árvores com DAP $\geq 40 \mathrm{~cm}$. Na picada de orientação foram fixadas estacas a cada $25 \mathrm{~m}$, nas quais foram afixadas placas com a metragem respectiva, o número da faixa e o da UT.

$\mathrm{Na}$ ficha de campo, além do registro da data, identificador, anotador, UT, faixa, ficha, azimute do caminhamento e informações quanto à variação topográfica e da vegetação, para o microzoneamento foram registradas as seguintes informações de cada árvore: número, nome vulgar, coordenadas $\mathrm{X}$ e $\mathrm{Y}$, CAP ou DAP, altura comercial, estado físico (viva, morta, em pé, caída, quebrada, oca etc.) e classe de qualidade do fuste (número de toras de $4 \mathrm{~m}$ ).

\section{Simulação do inventário amostral}

A largura das unidades de amostra testadas foi fixada em 50 metros, por ser a mesma utilizada para o censo florestal, a qual é aproximadamente o limite que um homem pode avistar na floresta para árvores de grande diâmetro. O comprimento das unidades de amostra variou de 50 a 400 metros, perfazendo uma área de 0,25 ha a 2,00 ha.

As unidades de amostra foram distribuídas de forma idêntica em cada unidade de trabalho de 100 ha e mantendo a distância mínima de 100 metros umas das outras, obedecendo ao limite estabelecido por Queiroz (1977).

Para os oito tamanhos de unidade de amostra, foram realizadas simulações com 10 unidades de amostra para cada tamanho e foi calculado o erro para a abundância. A intensidade de amostragem foi sendo ampliada em múltiplos de dez até que fosse atingido o limite mínimo desejado de erro. Uma vez cumprida a exigência com a variável abundância, selecionaram-se dois tamanhos para acurar a intensidade para a variável abundância e definir a intensidade para área basal e volume.

Consideraram-se como população comercial os indivíduos com DAP $\geq 40 \mathrm{~cm}$ pertencentes às espécies definidas pelo próprio setor empresarial, apresentadas na tabela 1.

Tabela 1. Lista de espécies componentes da população comercial da área de estudo.

Table 1. List of species component of the commercial population of the study area.

\begin{tabular}{lll}
\hline $\mathbf{N}$ & Nome vulgar & Nome científico \\
\hline 1 & Abiu/A.-Rosa & Pouteria sp. \\
2 & Açacu & Hura crepitans $\mathrm{L}$. \\
3 & Acariquara/Quariquara & Minquartia guianensis Aubl. \\
4 & Amarelão/Carapanaúba-branca & Aspidosperma sp. \\
5 & Angelim & Hymenolobium sp. \\
6 & Angico & Parkia sp. \\
7 & Bajão & Cassia sp. \\
8 & Bajinha & Stryphnodendron sp. \\
9 & Bálsamo/Cabreúva & Miroxylon balsamum (L.) Harms. \\
10 & Breu-branco & Protium sp. \\
11 & Caju & Anacardium giganteum Hanck ex Engl. \\
12 & Canela/Preciosa & Aniba canelilla (Kunth) Mez \\
13 & Catuaba & Qualea tesmanni Milldbr. \\
14 & Caucho/C.-amarelo & Castilla ulei Warb. \\
15 & Cedro/C.-rosa/C.-vermelho & Cedrela odorata L. \\
\hline
\end{tabular}




\begin{tabular}{|c|c|c|}
\hline 16 & Cerejeira/Cumaru-de-cheiro & Amburana acreana (Ducke) A. C. Sm. \\
\hline 17 & Copaíba & Copaifera sp. \\
\hline 18 & Corrimboque/Jequitibá & Cariniana sp. \\
\hline 19 & Cumaru/C.-ferro & Dipteryx odorata (Aubl.) Willd. \\
\hline 20 & Cumaru-cetim/Garapa & Apuleia molaris Spruce ex Benth. \\
\hline 21 & Currupixá/Abiu-da-casca-grossa & Micropholis melinoniana Pierre \\
\hline 22 & Farinha-seca & Celtis sp. \\
\hline 23 & Fava/F.-amarela & Pithecellobium sp. \\
\hline 24 & Fava-bajão & Parkia paraensis Ducke \\
\hline 25 & Fava-branca & Piptadenia sp. \\
\hline 26 & Fava-preta & Hymenolobium excelsum Ducke \\
\hline 27 & Figueira/F.-branca & Ficus sp. \\
\hline 28 & Freijó & Cordia alliodora (Ruiz e Pav.) Oken \\
\hline 29 & Guaribeiro & Phyllocarpus sp. \\
\hline 30 & Guariúba & Clarisia racemosa Ruiz \& Pav. \\
\hline 31 & Imbirindiba-preta & Terminalia sp. \\
\hline 32 & Ipê/Ipê-amarelo & Tabebuia serratifolia (Vahl) Nichols. \\
\hline 33 & Itaúba & Mezilaurus itauba (Meisn.) Taub. ex Mez \\
\hline 34 & Jacareúba & Calophyllum brasiliense Cambess. \\
\hline 35 & Jatobá & Hymenaea courbaril $\mathrm{L}$. \\
\hline 36 & Jequitibá & Cariniana estrellensis (Raddi) O. Ktze \\
\hline 37 & Jutaí & Hymenaea oblongifolia Huber \\
\hline 38 & Leiteira/Burra-leiteira & Sapium marmieri Huber \\
\hline 39 & Louro/L.-abacate & Ocotea sp. \\
\hline 40 & Macacaúba & Platymiscium trinitalis Benth \\
\hline 41 & Maçaranduba & Manilkara huberi (Ducke) Chevalier \\
\hline 42 & Manitê & Brosimum sp. \\
\hline 43 & Maparajuba & Pouteria sp. \\
\hline 44 & Maracatiara/Muiracatiara & Astronium lecointei Ducke \\
\hline 45 & Marfim/Pau-marfim & Agonandra brasiliensis Miers \\
\hline 46 & Marupá & Simarouba amara Aubl. \\
\hline 47 & Mirindiba/Imbirindiba/I.-amarela & Terminalia sp. \\
\hline 48 & Mogno & Swietenia macrophylla King. \\
\hline 49 & Pama & Pseudolmedia multinervis Mildbr. \\
\hline 50 & Pau-sangue & Pterocarpus amazonicus Huber \\
\hline 51 & Pereiro & Aspidosperma macrocarpon Mart. \\
\hline 52 & Pinho-cuiabano & Schizolobium amazonicum Huber ex Ducke \\
\hline 53 & Pintadinho & Tecophilaea procera C. Presl. \\
\hline 54 & Sorva & Couma macrocarpa Barb. Rodr. \\
\hline 55 & Sucupira/S.-amarela & Vatairea sericea Ducke \\
\hline 56 & Sumaúma/S.-branca & Ceiba pentandra (L.) Gaertn. \\
\hline 57 & Sumaúma-preta & Ceiba samauma K. Schum. \\
\hline 58 & Tamarindo & Dialium guianense (Aubl.) Sandwith \\
\hline 59 & Tauari & Couratari sp. \\
\hline 60 & Violeta & Platymiscium sp. \\
\hline
\end{tabular}

Os dados da amostragem foram testados considerando-se o objetivo de obter-se um erro de amostragem não superior a $10 \%$, com $95 \%$ de probabilidade de acerto.

Utilizou-se apenas a variável abundância para se definir o tamanho da unidade de amostra, até que o limite de $10 \%$ fosse obtido, testando-se posteriormente a amostragem para o volume e a área basal apenas para o tamanho da unidade de amostra selecionado.

A estatística dos dados foi processada como amostragem inteiramente casualizada, para população infinita ou finita, de acordo com a intensidade amostral. 


\section{RESULTADOS E DISCUSSÃO}

Considerando apenas o critério DAP $\geq 40 \mathrm{~cm}$ e a espécie, o Censo Florestal registrou 11.673 árvores das 60 espécies consideradas comerciais. Essa população adulta apresentou uma média de abundância de 11,7 árvores por ha, área basal de 4,04 $\mathrm{m}^{2}$ por ha e volume de $31,504 \mathrm{~m}^{3}$ por ha.

Com relação à simulação das amostragens, o limite de erro (E\%) foi atingido para abundância de indivíduos de DAP $\geq 40 \mathrm{~cm}$, com a unidade de amostra de 2,00 ha ou de 50 x $400 \mathrm{~m}$. A tabela 2 apresenta as estatísticas das unidades de amostra testadas.

Tabela 2. Resultados da amostragem em oito diferentes tamanhos de unidades de amostra.

Table 2. Results from the sampling in eight different sizes of sample units.

\begin{tabular}{|c|c|c|c|c|c|c|c|c|}
\hline \multirow{2}{*}{ Parâmetro } & \multicolumn{8}{|c|}{ Tamanho da Unidade de Amostra (em ha) } \\
\hline & $\mathbf{0 , 2 5}$ & $\mathbf{0 , 5 0}$ & $\mathbf{0 , 7 5}$ & $\mathbf{1 , 0 0}$ & 1,25 & $\mathbf{1 , 5 0}$ & 1,75 & 2,00 \\
\hline Área Total & 1000 & 1000 & 1000 & 1000 & 1000 & 1000 & 1000 & 1000 \\
\hline $\mathrm{N}$ & 60 & 60 & 60 & 60 & 60 & 60 & 60 & 60 \\
\hline $\mathrm{N}$ & 4000 & 2000 & 1333,33 & 1000 & 800 & 666,67 & 571,43 & 500 \\
\hline$(\mathrm{N}-\mathrm{n}) / \mathrm{N}$ & 0,985 & 0,97 & 0,955 & 0,94 & 0,925 & 0,91 & 0,895 & 0,88 \\
\hline Intensidade amostral (\%) & 1,5 & 3,0 & 4,5 & 6,0 & 7,5 & 9,0 & 10,5 & 12,0 \\
\hline Média da amostra & 3,03 & 6,37 & 9,03 & 11,28 & 14,12 & 16,90 & 19,02 & 21,97 \\
\hline Média por ha & 12,12 & 12,74 & 12,04 & 11,28 & 11,30 & 11,27 & 10,87 & 10,99 \\
\hline $\mathrm{S}_{\mathrm{x}}$ & 0,28 & 0,42 & 0,53 & 0,65 & 0,75 & 0,89 & 0,96 & 1,08 \\
\hline $\mathrm{T}(95 \%)$ & 2,0003 & 2,0003 & 2,0003 & 2,0003 & 2,0003 & 2,0003 & 2,0003 & 2,0003 \\
\hline $\mathrm{E}=\mathrm{T} \mathrm{S}_{\mathrm{x}}$ & 0,55 & 0,85 & 1,05 & 1,30 & 1,51 & 1,78 & 1,92 & 2,16 \\
\hline E \% & 18,28 & 13,27 & 11,67 & 11,51 & 10,67 & 10,55 & 10,12 & 9,85 \\
\hline $\mathrm{LI}=$ & 2,48 & 5,52 & 7,98 & 9,99 & 12,61 & 15,12 & 17,09 & 19,80 \\
\hline $\mathrm{LS}=$ & 3,59 & 7,21 & 10,09 & 12,58 & 15,62 & 18,68 & 20,94 & 24,13 \\
\hline $\mathrm{E}=\mathrm{LE}^{*} \times(10 \%)$ & 0,30 & 0,64 & 0,90 & 1,13 & 1,41 & 1,69 & 1,90 & 2,20 \\
\hline Dimensionamento $(\mathrm{n})^{*}$ & 18 & 43 & 67 & 78 & 68 & 66 & 61 & 58 \\
\hline Dimensionamento(ha)** & 4,61 & 21,43 & 50,05 & 77,91 & 84,51 & 99,19 & 107,21 & 116,78 \\
\hline $\mathrm{CV} \%$ & 70,78 & 51,40 & 45,21 & 45,95 & 42,96 & 42,83 & 41,41 & 40,65 \\
\hline Número de espécies & 40 & 46 & 50 & 50 & 51 & 51 & 51 & 51 \\
\hline
\end{tabular}

O erro amostral expresso em \% e o Coeficiente de Variação decresceram com o aumento do tamanho da unidade de amostra. Exceção apenas para o coeficiente de variação, que aumentou quando a unidade de amostra passou de 0,75 ha para 1,00 ha. A figura 1 apresenta o comportamento do Coeficiente de Variação, de acordo com o tamanho da unidade de amostra.

Observa-se na figura 1 que o coeficiente de variação tendeu a estabilizar a partir da unidade de amostra de 0,75 ha, o que indicaria a escolha desse tamanho. Entretanto, deslocamentos na floresta são dispendiosos e cansativos, aumentando consequentemente a probabilidade de ocorrência de erros nãoamostrais.

Observa-se na tabela 2 que os sistemas registraram no máximo 51 espécies, quando a população real é composta de 60 espécies.

Com relação à abundância estimada por cada amostragem simulada, a figura 2 ilustra a comparação de cada tamanho de unidade de amostra com a média verdadeira obtida no censo florestal.

Ainda de acordo com a figura 2, constata-se que a média verdadeira foi contemplada nos intervalos de confiança calculados. Constata-se também que, com unidades de amostra de até 0,75 ha, a média da população foi superestimada. Ao mesmo tempo, as unidades de amostra de tamanhos maiores do que 0,75 subestimaram a abundância da população.

Com 60 unidades de amostra, apenas com unidades de 2 ha obteve-se estimativa de média dentro do limite de erro requerido. Entretanto, o dimensionamento estimado para a unidade de amostra de 1 ha sugeriu que 78 unidades de amostra (ou 78 hectares) seriam suficientes para atingir o limite de erro requerido, ou seja, uma amostra $35 \%$ menor do que os 120 hectares que foram necessários para a unidade de amostra de 2,00 ha. 


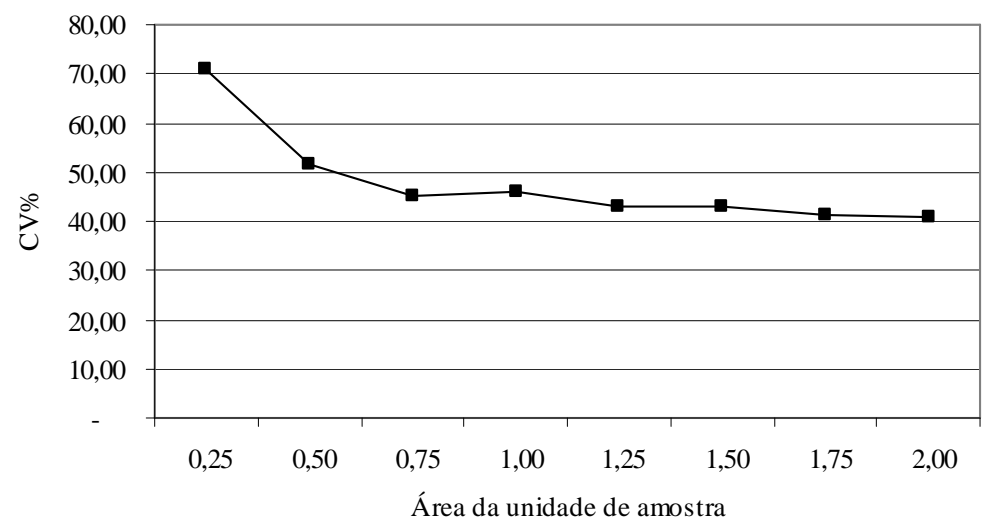

Figura 1. Comportamento do Coeficiente de Variação em função do tamanho da unidade de amostra. Figure 1. Behavior of the Coefficient of Variation as a function of the sample unit size.

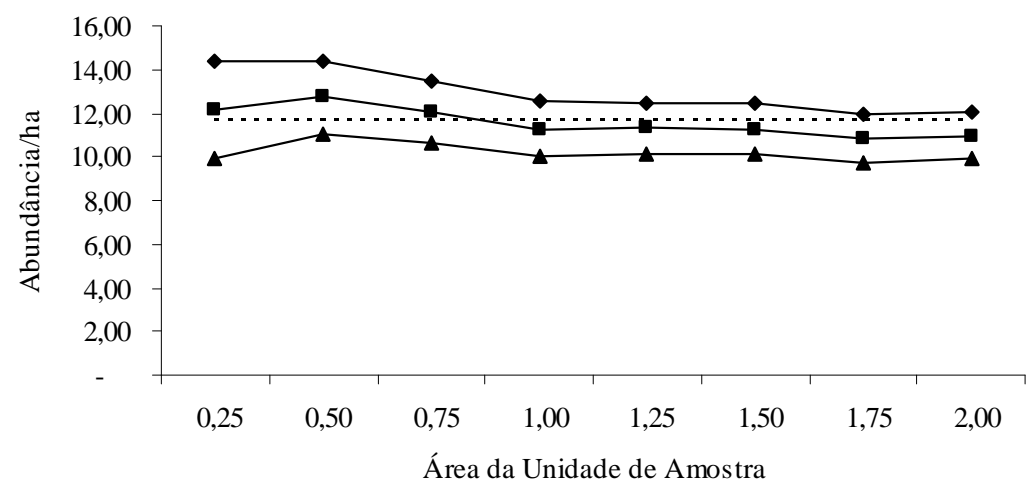

$\longrightarrow$ Estimada $\longrightarrow$ Limite inferior $\longrightarrow$ Limite superior $\cdots \cdots \cdot$ Real

Figura 2. Comparação da abundância média real com a média estimada, conforme a variação do tamanho das unidades de amostra.

Figure 2. Comparison of the observed average abundance with the estimate average, according to the variation of the sample units size.

Considerando o ponto de estabilidade do Coeficiente de Variação, o dimensionamento da amostra, estimado por meio do erro amostral, e a praticidade de se trabalhar com número inteiros múltiplos de 1 ha, optou-se por desenvolver as simulações com a unidade de amostra de 1,00 ha e de 2,00 ha. A tabela 3 apresenta o resultado das respectivas simulações.

Em relação à intensidade amostral calculada para a precisão requerida, o sistema com 1 ha levou vantagem na estimativa da abundância e da área basal, requerendo $8,5 \%$ e 10,7\%, respectivamente, contra $11,6 \%$ e $12,8 \%$ no sistema com unidades de amostra de 2 ha. Com relação ao volume, por sua vez, o sistema de 2 ha apresentou uma demanda de intensidade amostral ligeiramente menor, de 10,1 deste contra $10,3 \%$ do sistema de 1 ha.

Contudo, na avaliação da vantagem relativa do sistema de 1 ha deve ser considerada a quantidade de unidades de amostra necessárias para atingir a intensidade requerida, pois quanto maior o número de unidades de amostra, maior o custo de deslocamento das equipes de campo.

Considerando também que não serão implantadas parcelas para a avaliação de apenas uma variável, na prática, a variável mais exigente será a que determinará a intensidade amostral. Nesse caso, o 
sistema de 1 ha necessitará de 107 deslocamentos, e o sistema de 2 ha, de 64. No caso de uso de conglomerados, com 4 unidades amostrais, serão necessários 27 e 16 deslocamentos, respectivamente.

Tabela 3. Estatística da amostragem com unidades de amostra de 1 e 2 ha.

Table 3. Statistics of the sampling with sample units of 1 and 2 ha.

\begin{tabular}{|c|c|c|c|c|c|c|}
\hline \multirow{2}{*}{ Variável } & \multicolumn{3}{|c|}{ Unidade de 50 x $200 \mathrm{~m}$} & \multicolumn{3}{|c|}{ Unidade de 50 x $400 \mathrm{~m}$} \\
\hline & $\mathbf{A}$ & $\mathbf{G}$ & $\mathbf{V}$ & $\mathbf{A}$ & $\mathbf{G}$ & $\mathbf{V}$ \\
\hline Área Total (ha) & 1.000 & 1.000 & 1.000 & 1.000 & 1.000 & 1.000 \\
\hline Área da unidade de amostra (ha) & 1,00 & 1,00 & 1,00 & 2,00 & 2,00 & 2,00 \\
\hline $\mathrm{N}$ & 85 & 107 & 104 & 58 & 64 & 52 \\
\hline $\mathrm{N}$ & 1.000 & 1.000 & 1.000 & 500 & 500 & 500 \\
\hline$(\mathrm{N}-\mathrm{n}) / \mathrm{N}$ & 0,92 & 0,89 & 0,90 & 0,88 & 0,87 & 0,90 \\
\hline Intensidade amostral (em \%) & 8,5 & 10,7 & 10,4 & 11,6 & 12,8 & 10,4 \\
\hline Média da amostra & 11,5 & 3,76 & 29,996 & 22,2 & 7,26 & 62,827 \\
\hline Média por ha & 11,5 & 3,76 & 29,996 & 11,1 & 3,63 & 31,414 \\
\hline Sx & 0,6 & 0,19 & 1,519 & 1,1 & 0,37 & 3,077 \\
\hline $\mathrm{T}(95 \%)$ & 1,9714 & 1,9639 & 1,9649 & 2,0024 & 1,9785 & 2,0086 \\
\hline $\mathrm{E}=\mathrm{TS} \mathrm{x}$ & 1,1 & 0,38 & 2,985 & 2,2 & 0,72 & 6,181 \\
\hline $\mathrm{E} \%$ & 9,99 & 9,98 & 9,95 & 9,98 & 9,89 & 9,84 \\
\hline Limite inferior & 10,3 & 3,38 & 27,011 & 20,0 & 6,53 & 56,646 \\
\hline Limite superior & 12,6 & 4,13 & 32,980 & 24,4 & 7,98 & 69,008 \\
\hline $\mathrm{E}=\mathrm{LE} * \mathrm{x}(10 \%)$ & 1,1 & 0,38 & 3,000 & 2,2 & 0,73 & 6,283 \\
\hline Dimensionamento (n)* & 85 & 107 & 103 & 58 & 64 & 50 \\
\hline Dimensionamento $(\%) * *$ & 8,5 & 10,7 & 10,3 & 11,6 & 12,8 & 10,1 \\
\hline $\mathrm{CV} \%$ & 48,85 & 55,65 & 54,56 & 40,39 & 43,21 & 37,31 \\
\hline
\end{tabular}

A: Abundância em árvores; G: Área basal $\mathrm{em} \mathrm{m}^{2} ; \mathrm{V}$ : Volume $\mathrm{em} \mathrm{m}^{3}$; * Dimensionamento da intensidade amostral em número de unidades de amostra; ** Dimensionamento da intensidade amostral em hectares.

A figura 3 apresenta a comparação entre as estimativas transformadas para os valores reais, todos com média por ha. Uma vez transformados os valores em equivalentes a hectare, sob o ponto de vista das médias, os dois sistemas se aproximaram dos valores reais obtidos no censo florestal.

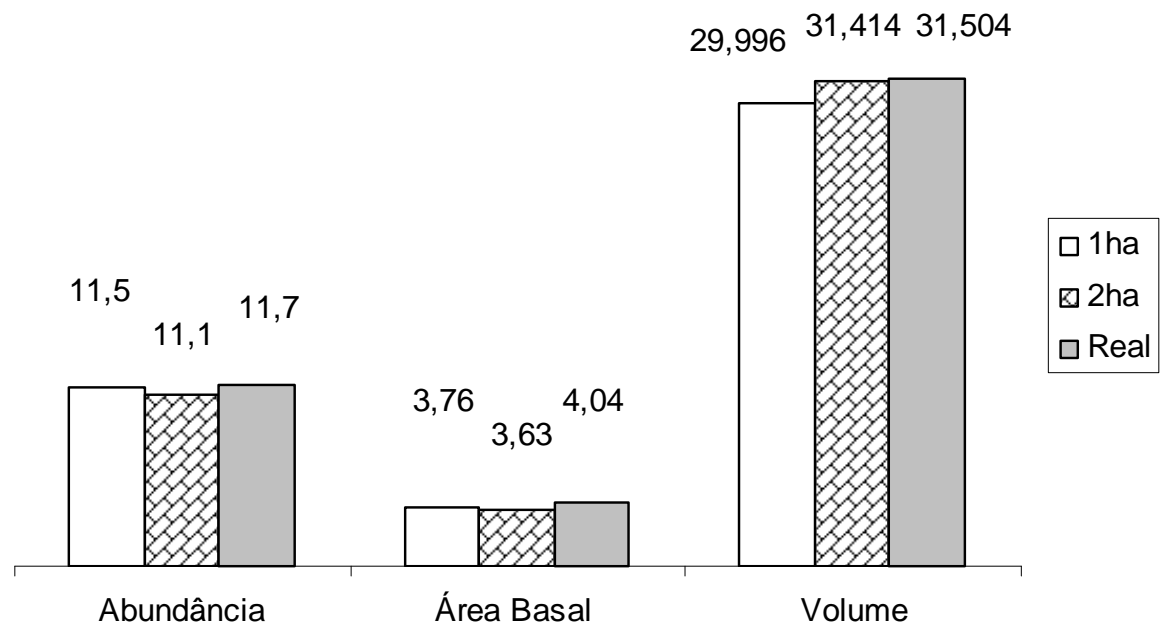

Figura 4. Comparação da abundância (árvores/ha), da área basal $\left(\mathrm{m}^{2}\right)$ e do volume $\left(\mathrm{m}^{3}\right)$ por ha entre os sistemas testados e os valores reais.

Figure 4. Comparison of the abundance (tree/ha), basal area $\left(\mathrm{m}^{2}\right)$ and volume $\left(\mathrm{m}^{3}\right)$ per ha between the tested systems and the true values. 


\section{CONCLUSÕES E RECOMENDAÇÕES}

Com base nos resultados, concluiu-se que o erro amostral em porcentagem e o coeficiente de variação se estabilizaram a partir do tamanho da unidade amostral de 0,75 ha, embora apresentem pequenas flutuações, chegando a um mínimo para tamanhos de 2 ha.

Os valores estimados por hectare da abundância, da área basal e do volume constituem estimativas bem próximas de seus respectivos valores verdadeiros, tanto para unidades amostrais de 1 ha como de 2 ha.

Uma vez que são três as variáveis de interesse, o número de unidades amostrais necessárias deve ser definido pela variável mais exigente, que no presente estudo foi área basal por hectare.

Embora não se tenha estimado o custo desses levantamentos, o custo dos deslocamentos necessários para o sistema de 1 ha, aliado aos demais parâmetros avaliados, sugere que o sistema com unidades de amostra de 2 ha seja mais adequado para atender às exigências estabelecidas.

Considerando-se o custo de inventários florestais por amostragem e a limitação das informações por eles geradas, é recomendável a desobrigação total do inventário amostral em florestas privadas, uma vez que as autorizações de exploração são baseadas no volume por espécie registrado no censo florestal.

No caso das florestas públicas, cujo direito de exploração será licitado, é recomendável delegar ao empresário concorrente e ao engenheiro florestal responsável pelo plano de manejo a decisão do investimento na intensidade da amostragem, que deverá ser tomada com base na análise de custo-benefício, uma vez que a autorização para exploração também será dada em função das informações do censo florestal.

Uma vez que a informação do inventário será a mesma para todos os concorrentes, é recomendável desobrigar as empresas de tal gasto, dando-lhes alternativa de acesso às áreas-objeto da concorrência e às informações existentes em cada caso.

A ampliação do limite de erro, no mínimo para $20 \%$ da média, ou a fixação do custo do inventário, através da implantação de um sistema padrão com amostragem sistemática parece ser a alternativa mais coerente com a atual exigência de limites de erro amostral.

\section{AGRADECIMENTOS}

À Cia. de Compensados Triunfo, do Mato Grosso e do Acre, ao Valteir Costa e ao Jáderson Mendes, pelas informações prestadas.

Ao Serviço Florestal Brasileiro, pelo apoio, com especial ênfase à engenheira florestal Anna Fanzeres.

\section{REFERENCIAS}

FUNTAC. Fundação de Tecnologia do Estado do Acre - FUNTAC. Relatório do Inventário Florestal Diagnóstico e Plano de Manejo da Fazenda São Jorge. Rio Branco, 2002.

HIGUCHI, N. Amostragem Sistemática Versus Amostragem Aleatória em Floresta Tropical Úmida. Acta amazônica, Manaus, v. 16/17, n. único, p. 393-400, 1987.

HIGUCHI, N.; SANTOS, J.; JARDIM, F. C. S. Tamanho de parcela amostral para inventários florestais. Acta Amazônica. Manaus, v. 12, n. 1, 91-103, 1982.

QUEIROZ, W. T. Análise de fatores (Facttor analysis) pelo método de máxima verossimilhança: aplicação ao estudo de estrutura de florestas tropicais. Piracicaba: ESALQ. 1984. 112p. Tese de Doutorado - ESALQ, 1984.

QUEIROZ, W. T. Efeitos da variação estrutural em unidades amostrais na aplicação do processo de amostragem por conglomerados nas florestas do Planalto do Tapajós. Curitiba: UFPR. 109 p. Dissertação de Mestrado - UFPR, 1977.

RADAMBRASIL. Folhas 19 Rio Branco; geologia, geomorfologia, pedologia, vegetação e uso potencial da terra: Volume 12. Departamento Nacional de Produção Mineral. 1976 Rio de Janeiro. $464 \mathrm{p}$.

SILVA, J. N. M. Eficiência de diversos tamanhos e formas de unidades de amostras aplicadas em inventário florestal na região do Baixo Tapajós, Curitiba: UFPR, 1980, 83p. Dissertação de Mestrado UFPR, 1980. 